\title{
Tratamientos químico y biológico en semillas de Aniba rosaeodora para el control de Heilipus odoratus (Coleoptera, Curculionidae, Molytinae) en viveros, Amazonas, Brasil
}

\author{
Chemical and biological treatments of seeds of Aniba rosaeodora for the control of Heilipus \\ odoratus (Coleoptera, Curculionidae, Molytinae) in greenhouses, Amazonas, Brazil
}

\author{
Adrian Arturo Arispe Torrez ${ }^{\text {a**, Larissa Ramos Chevreuil }}{ }^{\text {b }}$, Kally Alves de Sousa ${ }^{\text {c, }}$ \\ Paulo de Tarso Barbosa Sampaio d, Flávio Souza Bruno d, Ceci Sales-Campos b

\footnotetext{
*Autor de correspondencia: ${ }^{a}$ Universidad del Estado del Amazonas, Programa de Pos-Graduación en Biotecnología y Recursos Naturales, Av. Carvalho Leal n 1777, Manaos, Brasil, tel.: 5592 3878-4365, arturo.arispe.t@gmail.com

${ }^{\mathrm{b}}$ Instituto Nacional de Pesquisas de la Amazonia, Laboratorio de Cultivo de Hongos Comestibles, Manaos, Brasil.

c Instituto Federal de Educación, Ciencia y Tecnología de Rondonia (IFRO), Guajará-Mirim, Brasil.

d Instituto Nacional de Pesquisas de la Amazonia, Laboratorio de Propagación de Plantas y Tecnología Digital, Manaos, Brasil.
}

\begin{abstract}
SUMMARY
Aniba rosaeodora is a species of high international commercial value due to the essential oil in the wood, branches and leaves. Studies indicate problems in the annual production of fruits and seeds, due to intense insect attack, which is a limiting factor in the production of seedlings. The present study evaluates the efficiency of the insecticide (Karate Zeon 50 CS) and the extract of an entomopathogenic fungus Beauveria bassiana on the control of the larva of Heilipus odoratus in seeds and their influence on the initial development of the seedlings. In the first experiment, seeds were collected from the forest floor and tree canopy, later they were immersed in a liquid solution of $5 \mathrm{~mL} \mathrm{~L}^{-1}$ Karate Zeon insecticide for 24, 48 and 72 hours. In the second experiment, the seed were immersed in a concentration of $10^{6}$ conidia $\mathrm{mL}^{-1}$ of $B$. bassiana and incubated in an orbital mixer for 3 hours at $180 \mathrm{rpm}$ at $30{ }^{\circ} \mathrm{C}$. To continue, the seeds were collected to be germinated in a substrate of vermiculite $\left(28 \pm 3{ }^{\circ} \mathrm{C}\right)$ for 60 days; afterward, they were placed in $1 \mathrm{~kg}$ plastic bags and grown in a greenhouse with $(50 \%)$ shade and a daily mist for 270 days. The parameters evaluated were: percentage of germination $(\mathrm{G} \%)$, index of germination velocity ( $\mathrm{IGV})$, average germination time (AGT), growth, biomass (root, stem and leaves), chloroplast pigments content and leaf gas exchange. Results indicate that the treatments of seeds diminished the damage caused by the larva of $H$. odoratus increasing the index of germination without affecting the initial development.
\end{abstract}

Key words: biological control, germination, biomass, chloroplast pigments, gas exchange.

\section{RESUMEN}

Aniba rosaeodora es de gran valor comercial por su aceite esencial en la madera, ramas y hojas. Estudios indican problemas de fructificación anual y las semillas sufren intenso ataque de insectos, factor limitante en la producción de plántulas. El presente trabajo evaluó la eficiencia del insecticida (Karate Zeon 50 CS) y del extracto del hongo entomopatógeno Beauveria bassiana sobre el control de larvas de Heilipus odoratus en semillas y su influencia en el desarrollo inicial de las plántulas. En el primer experimento, semillas colectadas de la copa y del suelo, fueron inmersas en una solución de $5 \mathrm{~mL} \mathrm{~L}^{-1}$ de Karate Zeon, durante 24,48 y 72 horas. En el segundo experimento, las semillas colectadas en el suelo fueron inmersas en un medio líquido de concentración $10^{6}$ conidios $\mathrm{mL}^{-1}$ de B. bassiana e incubados en agitador orbital durante 3 horas a $180 \mathrm{rpm}$ y $30^{\circ} \mathrm{C}$. Posteriormente, las semillas fueron colocadas para germinar en sustrato vermiculita $\left(28 \pm 3{ }^{\circ} \mathrm{C}\right)$ durante 60 días, luego repicadas en contenedores plásticos de $1 \mathrm{~kg}$ y cultivadas en vivero dotado de tela de protección $(50 \%)$ con nebulización diaria durante 270 días. Los parámetros evaluados fueron: porcentaje de germinación (G \%), índice de velocidad de germinación (IVG), tiempo medio de germinación (TMG), crecimiento, biomasa (raíz, tallo y hojas), contenido de pigmentos cloroplastos e intercambio gaseoso foliar. Los resultados revelaron que el tratamiento de semillas detuvo los daños causados por larvas de H. odoratus aumentando los índices de germinación sin afectar el desarrollo inicial.

Palabras clave: control biológico, germinación, biomasa, clorofila, intercambio gaseoso.

\section{INTRODUCCIÓN}

Aniba rosaeodora Ducke es económicamente valorizada por el aceite esencial, extraído del fuste, ramas y hojas, utilizado comercialmente como fijador por la industria de perfumes (Krainovic et al. 2017). Esta especie se encuentra en peligro de extinción, presenta problemas de fructificación irregular y alta depredación de frutos $(59,5 \%)$ por 
aves e insectos de la orden Coleoptera y Lepidoptera (Spironello et al. 2004). La plaga principal identificada como Heilipus odoratus Vanin et Gaiger, 2005 (Coleoptera, Curculionidae, Molytinae) inicia el ataque cuando las hembras adultas perforan los frutos y comienzan ovopositar, en ese proceso, las larvas de este escarabajo se desenvuelven dentro de las semillas, alimentándose de las reservas de los cotiledones y cuando alcanzan el embrión interrumpen el proceso de germinación (Vanin y Gaiger 2005). Estos insectos pueden inducir la pérdida de viabilidad de los frutos, pues, la infestación es mayor en aquellos que fueron liberados en relación a frutos que permanecen en la copa de los árboles. La reunión de estos factores convierte la disponibilidad de material biológico en uno de los aspectos más difíciles de la propagación de $A$. rosaeodora.

Determinados insecticidas pueden proteger las semillas durante el proceso de germinación, específicamente, la mezcla de fipronil y acetamiprid en forma de saches, colocados en el sustrato de germinación, consiguió reducir los ataques de las semillas de $A$. rosaeodora causados por $H$. odoratus, obteniendo mayor número de plántulas germinadas (Morais et al. 2009). Por otro lado, el uso intensivo de insecticidas puede generar consecuencias nocivas, contaminando agua, suelo y aire, como también, algunos patógenos y plagas pueden desarrollar mayor resistencia a ciertos principios activos (Leite et al. 2006). En ese sentido, el principio activo de algunos insecticidas pueden influenciar negativamente la germinación y la sobrevivencia de plántulas, entretanto, pueden disminuir la depredación y la herbívora y así favorecer el desarrollo inicial (Barros et al. 2001).

El control biológico surge como un tratamiento alternativo de combate a los insectos-plagas, mostrándose altamente eficiente y menos perjudicial a la salud humana y al medio ambiente (Delgado y Muricia-Ordoñez 2011). El control microbiano de plagas en semillas consiste en la utilización de hongos entomopatógenos, destacándose Beauveria bassiana (Bals.) Vuillemin (Hyphomycete), presentando algunas ventajas específicas de selectividad (Ferreira et al. 2014). Entre las estrategias del control biológico, se utiliza el contacto y/o adherencia del microorganismo al tegumento externo de la semilla, aplicación foliar e inoculación en el suelo (Kaewkham et al. 2016).

Ante la falta de estudios direccionados al control del depredador de semillas (H. odoratus), es necesario aplicar técnicas de reducción de ataques en condiciones de laboratorio, pudiendo ser una alternativa los tratamientos químicos y/o biológicos. Sin embargo, poco se conoce sobre la influencia de estos tratamientos sobre las alteraciones morfológicas y fisiológicas en $A$. rosaeodora. Generalmente, estas alteraciones se expresan a través de los cambios en el área foliar, biomasa de las raíces, contenido de pigmentos cloroplastos y variables relacionadas a las características fotosintéticas (Gonçalves et al. 2005). Por ese motivo, es importante analizar los datos fisiológicos de las plántulas originadas de diferentes tratamientos, además de considerar los factores abióticos como agua, luz y temperatura, que influencian directamente en las reacciones bioquímicas de los metabolismos primario y secundario de las plantas (Gonçalves et al. 2016).

En ese sentido, se plantea como hipótesis que el tratamiento químico y/o biológico en semillas infectadas pueda controlar el desarrollo interno de la plaga H. odoratus, aumentando los índices de germinación sin afectar la fisiología de las plántulas. El presente trabajo tiene como objetivo evaluar la eficiencia del tratamiento de semillas con insecticida Karate Zeon 50 CS y con el hongo entomopatógeno Beauveria bassiana en el control de las larvas de $H$. odoratus y analizar posibles influencias en el desarrollo inicial de las plántulas.

\section{MÉTODOS}

Colectas del material biológico. De acuerdo al calendario fenológico de A. rosaeodora, en el estado del Amazonas, la época de floración se concentra en mayo y julio, posteriormente, los frutos comienzan a madurar en el mes de septiembre (Lorenzi 1998). Los frutos fueron colectados entre los meses de octubre y noviembre del 2016, de la copa de los árboles y del suelo alrededor de cinco matrices, localizados en la Reserva Forestal Adolpho Ducke Instituto Nacional de Pesquisas de la Amazonia (INPA), Manaos, Amazonas, Brasil. El clima es ecuatorial húmedo con promedio de precipitación anual de $2.286 \mathrm{~mm}$, temperatura promedio de $27,7^{\circ} \mathrm{C}$ y humedad relativa promedio del aire de 72,69\% (INMET 2017).

Posteriormente, los frutos fueron transportados al Laboratorio de Propagación de Plantas (INPA), donde los frutos inmaduros fueron colocados sobre papel de periódico a temperatura ambiente $\left(28 \pm 3{ }^{\circ} \mathrm{C}\right)$ hasta obtener la coloración violeta, y seguidamente fueron separados todos aquellos con indicios de ataques por larvas de $H$. odoratus (figura 1). Para facilitar la retirada de la pulpa, los frutos fueron inmersos en agua durante 48 horas. Luego, los ensayos experimentales fueron conducidos y evaluados según las Reglas para Análisis de Semillas (Brasil 2009).

Tratamiento químico. Semillas provenientes de la colecta de la copa del árbol $(n=400)$ y del suelo $(n=400)$ fueron subdivididas en dos grupos: a) 200 con tegumento b) 200 sin tegumento. Ambas fueron inmersas en una solución del insecticida Karate Zeon 50 CS (Syngenta Limited) de $5 \mathrm{~mL} \mathrm{~L}^{-1}$ durante 24,48 y 72 horas. El control consistió en semillas inmersas apenas en agua destilada por 72 horas, siendo un delineamiento enteramente aleatorizado con dos repeticiones de 25 semillas en bandeja plástica con sustrato vermiculita.

Tratamiento Biológico. Semillas colectadas en el suelo de las matrices fueron colocadas en contacto con el hongo entomopatógeno B. bassiana, aislado del suelo, gentilmente cedido por la colección del Laboratorio de Cultivo de Hongos Comestibles (LCFC) y activado en medio agar 
papa dextrosa (APD). En el preparo de las suspensiones, el hongo $B$. bassiana fue cultivado en tubos de ensayo en medio APD e incubado a $30{ }^{\circ} \mathrm{C}$ durante 10 días. Las suspensiones de hongos fueron preparadas adicionándose $10 \mathrm{~mL}$ de Tritón X-100 (BioAgency) estéril ( $0,1 \% \mathrm{v} / \mathrm{v})$ a los tubos de ensayo con el hongo en fase de crecimiento. Los conidios fueron raspados del medio de cultura con auxilio de alza de platino y homogenizado en el agitador para tubos Vortex (K40-1010). La determinación de la concentración de conidios fue realizada por medio de conteo en cámara de Neubauer para ajuste de $10^{8}$ conidios $\mathrm{mL}^{-1}$.

Los conidios $\left(10^{8}\right.$ conidios $\left.\mathrm{mL}^{-1}\right)$ fueron inoculados en medio líquido ( $20 \mathrm{~g}$ de glucosa y $20 \mathrm{~g}$ de extracto de levadura para el volumen final de $1 \mathrm{~L}$ ) e incubados en agitador orbital a $220 \mathrm{rpm}, 30^{\circ} \mathrm{C}$ durante 72 horas. La concentración final de conidios en el medio líquido fue $10^{6}$ conidios $\mathrm{mL}^{-1}$. Después de 72 horas, las semillas fueron inmersas en medio líquido conteniendo los micelios desarrollados de B. bassiana y agitados a $180 \mathrm{rpm}$, en temperatura de $30^{\circ} \mathrm{C}$ durante 3 horas.

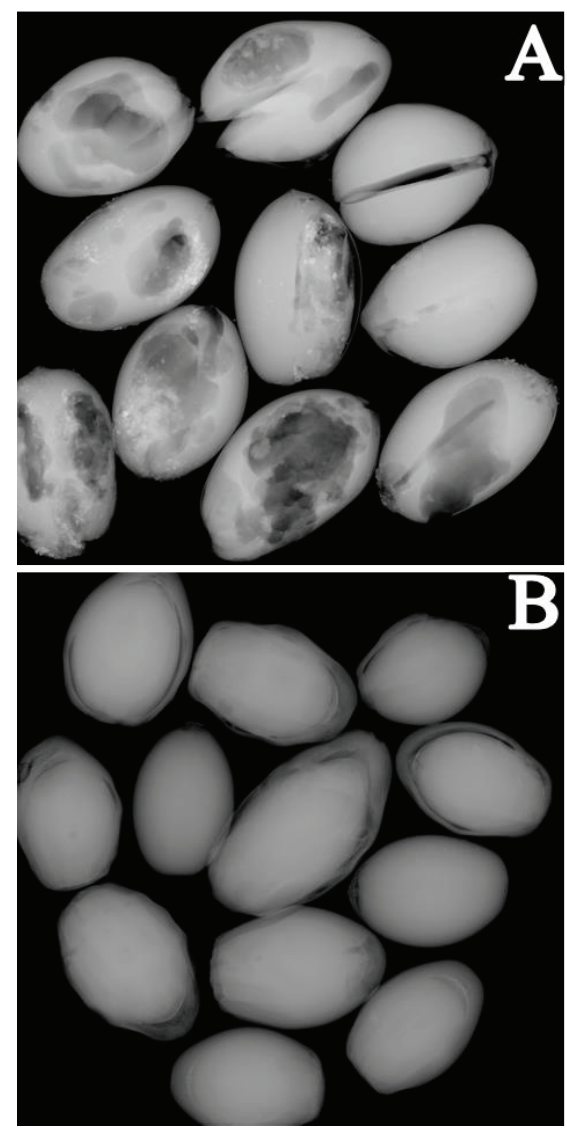

Figura 1. Imagen de placa de rayos-X de semillas de Aniba rosaeodora Ducke. A) Semillas atacadas por larvas de H. odoratus. B) Semillas en óptimo estado fitosanitario sin indicio de ataques.

X-ray image of Aniba rosaeodora seeds. A) Seeds attacked by larva $H$. odoratus. B) Seeds in a good phytosanitary state without indication of attack.
Fueron seleccionadas 80 semillas con tegumento y 80 sin tegumento, colocadas en bandejas plásticas con sustrato vermiculita en casa de vegetación, con temperatura ambiente de $28 \pm 3{ }^{\circ} \mathrm{C}$. Como control fueron usadas semillas en contacto con el medio líquido de propagación del hongo, sin la presencia de B. bassiana. El delineamiento experimental utilizado fue enteramente aleatorizado, con cuatro repeticiones de 20 semillas por tratamiento, siendo cada grupo control compuesto de dos repeticiones de 20 semillas.

Análisis de germinación. Durante 60 días, fueron evaluados los siguientes parámetros: a) porcentaje de germinación $(\mathrm{G} \%)$, tiempo medio de germinación (TMG), índice de velocidad de germinación (IVG) y tiempo medio de emergencia (TME).

Desarrollo inicial. A los 90 días, las plántulas fueron repicadas para sacos plásticos de $1 \mathrm{~kg}$ conteniendo sustrato obtenido de la camada superior del suelo $(10-30 \mathrm{~cm})$ del INPA con clasificación latosólico rojo de textura arcillosa (Embrapa 2006) y transportados al vivero de crecimiento dotado de tela de protección (50 \%) contra los rayos solares y sistema de nebulización, obedeciendo la siguiente disposición: grupo control, grupo 1 (tratamiento químico) y grupo 2 (tratamiento biológico).

Los análisis biométricos fueron realizados con muestras de 50 plántulas por tratamiento, midiéndose altura total y diámetro del tallo con parquímetro digital (Mitutoyo) a los 150, 210 y 270 días. En cada evaluación fueron seleccionadas cinco plántulas por tratamiento, separándose hoja, tallo y raíces para ser digitalizadas en el software TinyScanner. Para determinar el área foliar, las imágenes de las hojas fueron analizadas en el software Imagej.

Posteriormente, para determinación de la biomasa de hojas, tallos y raíces, las muestras fueron pesadas en balanza de precisión (Shimadzu-Clase II) y secadas en estufa (Tecnal TE-394/3) con temperatura constante de $63 \pm 3{ }^{\circ} \mathrm{C}$ durante 72 horas. A continuación, fue obtenido el peso seco de las muestras y el área foliar especifica (AFE) fue calculada dividiendo el área foliar $\left(\mathrm{cm}^{2}\right)$ entre la masa seca de las hojas (g) de cada plántula. La razón de área foliar (RAF) fue calculada dividiendo el área total de las hojas entre la masa seca de la plántula (Green y Newbery 2001).

El intercambio gaseoso fue evaluado a través de las medidas de fotosíntesis líquida (A), respiración en lo oscuro (Rd), transpiración (E) y conductancia estomática (gs), con apoyo de un analizador de gas infrarrojo portátil (IRGA), de sistema abierto, modelo LI-COR 6400, conforme a la metodología para especies amazónicas (Marenco et al. 2001). Los datos fueron colectados a los 240 días de edad, entre 9:00 y 12:00 horas de forma aleatoria, siendo las lecturas realizadas en cuatro plántulas por tratamiento (control, grupo 1 y grupo 2). Los valores puntuales de intercambio gaseoso fueron obtenidos con cámara foliar ajustada para irradiación en torno de $1.000 \mu \mathrm{mol} \mathrm{m}^{-2} \mathrm{~s}^{-1}$. El IRGA 
fue ajustado bajo concentración de $400 \pm 10 \mu \mathrm{mol} \mathrm{mol}^{-1}$ de $\mathrm{CO}_{2}$, temperatura de $31 \pm 1^{\circ} \mathrm{C}$ y vapor de $\mathrm{H}_{2} \mathrm{O}$ de $23 \pm$ $1 \mathrm{mmol} \mathrm{mol}^{-1}$. La respiración en lo oscuro $\left(\mathrm{R}_{\mathrm{d}}\right)$ fue determinada después del período de adaptación de 10 minutos, con la cámara ajustada para irradiación de $0 \mu \mathrm{mol} \mathrm{m}^{-2} \mathrm{~s}^{-1}$. La eficiencia en el uso de agua (EUA mmol $\mathrm{CO}_{2} \mathrm{~mol}^{-1} \mathrm{H}_{2} \mathrm{O}$ ) fue calculada por la relación fotosíntesis/transpiración.

Los tenores de pigmentos cloroplastos fueron determinados a partir de las hojas completamente expandidas y en buen estado fitosanitario, seleccionándose aleatoriamente 10 plántulas de cada tratamiento. Inicialmente, fueron realizadas lecturas con el medidor de clorofila (SPAD-502), en cinco puntos a cada lado de la nervadura central sobre el haz de la hoja. Posteriormente, las hojas fueron colectadas y acondicionadas en recipientes con agua destilada, visando evitar la deshidratación foliar, luego inducidas a la determinación del contenido de clorofila $a, b$ y carotenoides. Para eso, se retiró 10 discos de $6 \mathrm{~mm}$ de diámetro/hoja para la extracción de los pigmentos cloroplastos de acuerdo con el método de Arnon (1949), utilizándose acetona $80 \%$ $\left(10 \mathrm{~mL}\right.$ muestra $\left.{ }^{-1}\right)$, cuarzo $\left(10 \mathrm{~mL}\right.$ muestra $\left.{ }^{-1}\right)$ y $\mathrm{MgCO}_{3}$ $\left(10 \mathrm{mg} \mathrm{muestra}^{-1}\right)$. El material foliar fue triturado y filtrado en papel cuantitativo $(125 \mathrm{~mm})$ e inmediatamente realizadas las lecturas espectrofotométricas a $663 \mathrm{~nm}$ (clorofila $a$ ), $645 \mathrm{~nm}$ (clorofila $b$ ) e $480 \mathrm{~nm}$ (carotenoides). Las concentraciones de los pigmentos cloroplastos fueron determinadas a partir de las ecuaciones descritas por Hendry y Price (1993):

$$
\begin{gathered}
\text { Chl } a=\frac{\left(12,7 * \mathrm{~A}_{663}-2,69 * \mathrm{~A}_{645}\right) * 1,119 * \text { Volumen }}{1000 * \text { peso }(\mathrm{g})} \\
\text { Chl } b=\frac{\left(22,9 * \mathrm{~A}_{645}-4,68 * \mathrm{~A}_{663}\right) * 1,102 * \text { Volumen }}{1000 * \text { peso }(\mathrm{g})} \\
\mathrm{C}_{x+c}=\frac{\left(\mathrm{A}_{480}+0,114 * \mathrm{~A}_{663}-0,638 * \mathrm{~A}_{645}\right) * \text { Volumen*1000 }}{112,5 * \text { peso }(\mathrm{g})}
\end{gathered}
$$

Donde, Chl $a=$ Clorofila $a$. Chl $b=$ Clorofila $b . \mathrm{C}_{\mathrm{x}+\mathrm{c}}=\mathrm{Ca}-$ rotenoides.

A partir de las concentraciones de clorofilas y carotenoides fueron calculadas las concentraciones de clorofila total $(C h l a+C h l b)$, la razón de clorofila $a /$ clorofila $b(C h l$ $a / b)$ y clorofila total/carotenoides $\left(C h l\right.$ total $\left./ \mathrm{C}_{\mathrm{x}+\mathrm{c}}\right)$.

Los datos obtenidos se sometieron a análisis de varianza (ANDEVA) y prueba de Tukey $(P \leq 0,05)$ para comparación de medias. El análisis de datos fue ejecutado con el programa Assistat 7.7 y las figuras realizadas con SigmaPlot 13.0.

\section{RESULTADOS}

Germinación del tratamiento químico. La germinación comenzó a partir del $23^{\circ}$ día. La comparación entre colectas tuvieron diferencias significativas $(\mathrm{F}=37,78 ; P<0,01)$, siendo las semillas colectadas de la copa del árbol las que presentaron un mayor índice de germinación (78 \%), superior a las que fueron colectadas del suelo $(63,47 \%)$. En ese sentido, la inter-relación (colectas y semillas) determinó que la presencia del tegumento reduce la germinación $(\mathrm{F}=76,37 ; P<0,01)$ en las dos colectas (figura 2).

La interacción entre el periodo de imbibición (control, 24,48 y 72 horas) y las colectas (copa, suelo) demuestra que existió diferencia significativa $(\mathrm{F}=14,81 ; P<0,01)$. La germinación de las semillas de la copa del árbol fue aumentando gradualmente durante los periodos de 24, 48 y 72 horas, sin embargo, no tuvieron diferencias entre los tratamientos, por eso, se determinó que 24 horas es ideal para el control químico de las semillas (cuadro 1). Con relación a los periodos de imbibición de las colectas en el suelo, el tratamiento control no varió entre los periodos de 24 y 48 horas, sin embargo, la germinación del periodo de 72 horas fue pérdida $(50,58 \%)$ en relación al control y tratamientos.

El TMG presentó diferencias significativas entre las colectas $(\mathrm{F}=428,61 ; P<0,01)$, las que fueron recogidas del suelo consiguieron germinar más rápido (aprox. 29 días) cuando fueron comparadas con las adquiridas de

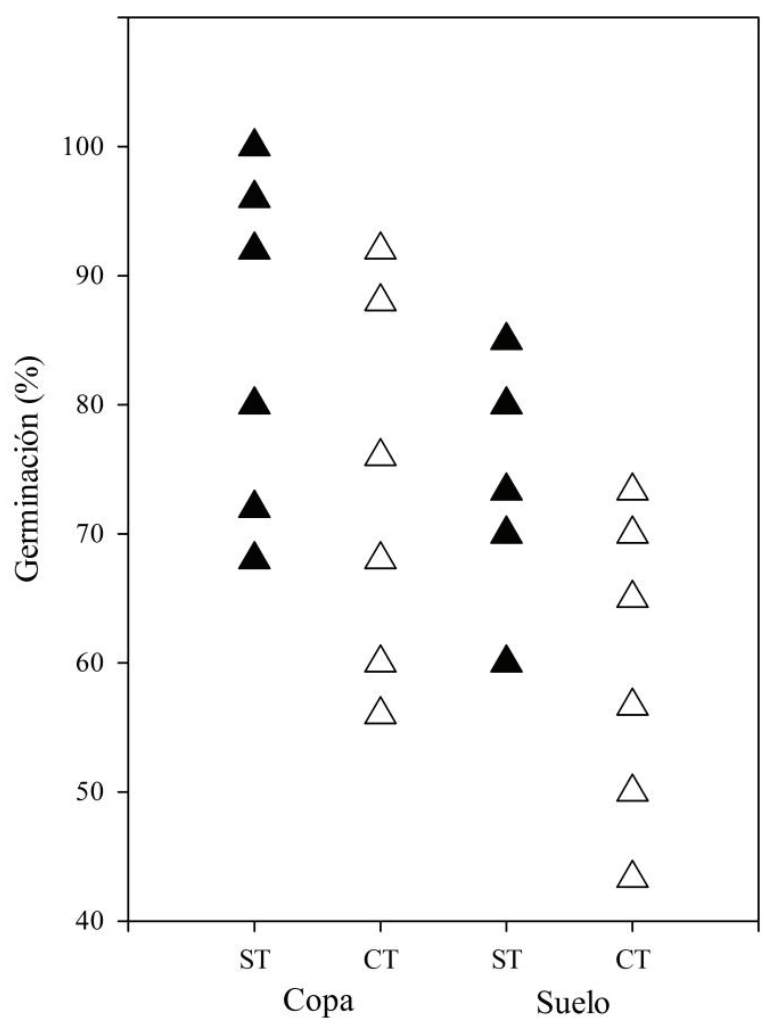

Figura 2. Germinación de las semillas de Aniba rosaeodora procedentes de dos tipos de colectas, subdivididas en: sin tegumento (ST) y con tegumento (CT).

Germination of the seeds of Aniba rosaeodora from two types of collections subdivided in: without tegument and with tegument. 
la copa del árbol $(\approx 36$ días). El IVG presentó diferencias en las colectas ( $\mathrm{F}=428,61 ; P<0,01)$, por otro lado, las semillas de la copa de los árboles presentaron el mayor promedio de IVG $(\mathrm{F}=61,67 ; P<0,01)$, en los tratamientos de 24 y 48 horas, sin embargo, las semillas recogidas del suelo, no tuvieron diferencia alguna entre los tratamientos.

Germinación de tratamiento biológico. La germinación de las semillas con y sin tegumento (CT, ST) colocadas en contacto con el hongo entomopatógeno $B$. bassiana, tuvo inicio a partir de 20 días. El contacto con el hongo aumentó los índices de germinación en relación al control, sin embargo, al igual que el TMG e IVG no obtuvo diferencias significativas, entre tanto, el TME de semillas sin tegumento, aceleró el proceso de germinación, disminuyendo de 41 para 28 días (cuadro 2).

Desarrollo inicial. El crecimiento de plántulas de $A$. rosaeodora fue proporcional en los periodos de medición, inició a los 120 días con $10-15 \mathrm{~cm}$ y a los 270 días alcanzó entre 32 y $35 \mathrm{~cm}$ de altura, sin embargo, los valores medios de altura, diámetro del tallo y número de hojas de los tratamientos, determinaron que las plántulas mantienen uniformidad en el desarrollo inicial en relación al control (cuadro 3).

Biomasa. De acuerdo a las evaluaciones de la masa seca de las raíces y de la parte aérea, el tratamiento de semillas no influenció la cantidad de materia retenida en las hojas y la materia seca acumulada en toda la planta (cuadro 4). El área foliar fue incrementando con el tiempo, lo que per- mitió observar un óptimo desarrollo en relación al control, que a los 150 días presentaban $157,70 \mathrm{~cm}^{2}$ y alcanzó los $479,05 \mathrm{~cm}^{2}$ a los 270 días de evaluación. A los 150 días fue realizada la primera medición y la razón de área foliar (RAF) y área foliar especifica (AFE) indicaron que el grupo control no presentó diferencia en relación al grupo 1 y 2 , siendo que el grupo 2 fue mayor al grupo 1 . En las siguientes mediciones, las variables mantuvieron un padrón similar.

Cuadro 2. Parámetros de la germinación de semillas de Aniba rosaeodora con tratamiento biológico de Beauveria bassiana.

Parameters seed germination of Aniba rosaeodora with biological treatment by Beauveria bassiana.

\begin{tabular}{lccc}
\hline \multicolumn{1}{c}{ Parámetros ${ }^{\mathrm{ns}}$} & Semillas & Control & Imbibición \\
\hline Germinación (\%) & ST & 59,99 & 73,97 \\
& CT & 61,00 & 75,59 \\
\hline $\begin{array}{l}\text { Tiempo medio de } \\
\text { germinación (días) }\end{array}$ & ST & 22,24 & 23,06 \\
\hline Índice de velocidad & ST & 33,49 & 35,69 \\
de germinación (días) & CT & 0,26 & 0,23 \\
\hline $\begin{array}{l}\text { Tiempo medio de } \\
\text { emergencia (días) }\end{array}$ & ST & 27,43 & 0,15 \\
\hline
\end{tabular}

ns $=$ No significativo.

$n s=$ Not significant

Cuadro 1. Interacción entre las colectas de semillas y los tiempos de imbibición en insecticida químico y su influencia sobre la germinación de Aniba rosaeodora.

Interaction between seed collection and imbibition times in chemical insecticide and its influence on seed germination of Aniba rosaeodora.

\begin{tabular}{cccccc}
\hline \multirow{2}{*}{ Parámetros } & Colectas & \multicolumn{4}{c}{ Tiempos de imbibición* } \\
\cline { 3 - 6 } & & Control & $24 \mathrm{~h}$ & $48 \mathrm{~h}$ & $72 \mathrm{~h}$ \\
\hline \multirow{2}{*}{ Germinación (\%) } & Copa & $63,0 \mathrm{aB}$ & $80,0 \mathrm{aA}$ & $83,0 \mathrm{aA}$ & $86,0 \mathrm{aA}$ \\
& Suelo & $71,6 \mathrm{aA}$ & $67,0 \mathrm{bA}$ & $64,5 \mathrm{bA}$ & $50,6 \mathrm{bB}$ \\
\hline $\begin{array}{c}\text { Tiempo medio de } \\
\text { germinación (días) }\end{array}$ & Copa & $38,7 \mathrm{bB}$ & $38,0 \mathrm{bB}$ & $34,1 \mathrm{bA}$ & $32,0 \mathrm{bA}$ \\
\hline $\begin{array}{c}\text { Índice de velocidad de } \\
\text { germinación (días) }\end{array}$ & Suelo & $27,3 \mathrm{aA}$ & $31,2 \mathrm{aB}$ & $29,1 \mathrm{aAB}$ & $27,8 \mathrm{aAB}$ \\
\hline $\begin{array}{c}\text { Tiempo medio de } \\
\text { emergencia (días) }\end{array}$ & Suelo & $0,46 \mathrm{aC}$ & $0,59 \mathrm{aB}$ & $0,66 \mathrm{aAB}$ & $0,73 \mathrm{aA}$ \\
\hline
\end{tabular}

*Diferentes letras en cada columna, minúsculas para las colectas y mayúscula para los tiempos de imbibición, indican diferencias significativas (Tukey, $P<0,05)$.

Different letters in each column, uppercase for collection and lowercase for time of soaking, indicate differences of significance (Tukey, $P<0.05$ ). 
Intercambio gaseoso. Los valores de la fotosíntesis liquida y transpiración entre el control y el grupo 1 fueron iguales estadísticamente, entre tanto, al ser comparadas con las plántulas del grupo 2, presentaron variaciones, indicando que la fotosíntesis y valor de la transpiración fue menor $\left(3,70 \mu \mathrm{mol} \mathrm{CO} \mathrm{m}^{-2} \mathrm{~s}^{-1} ; 0,78 \mathrm{mmol}_{2} \mathrm{O} \mathrm{m}^{-2} \mathrm{~s}^{-1}\right.$, respectiva- mente) cuando comparadas con el control. Los valores de respiración en lo oscuro son iguales entre los tratamientos, como también, la conductancia estomática. Por otro lado, la eficiencia en el uso de agua presentó diferencias significativas, siendo que el grupo $1 \mathrm{y}$ el control fueron iguales, diferenciándose del tratamiento biológico (cuadro 5).

Cuadro 3. Evaluación del crecimiento de plántulas de Aniba rosaeodora originadas de los tratamientos químico (grupo 1) y biológico (grupo 2) a los 150, 210 y 270 días.

Evaluation of the growth of seedlings of Aniba rosaeodora, originated from the chemical treatment (group 1) and biological treatment (group 2) at 150, 210 and 270 days.

\begin{tabular}{|c|c|c|c|c|c|c|c|}
\hline \multirow{2}{*}{$\begin{array}{l}\text { Edad } \\
\text { (días) }\end{array}$} & \multirow{2}{*}{ Tratamientos } & \multicolumn{2}{|c|}{ Altura } & \multicolumn{2}{|c|}{ Diámetro del tallo } & \multicolumn{2}{|c|}{$\mathrm{N}^{\circ}$ Hojas } \\
\hline & & $(\mathrm{cm})$ & CV (\%) & $(\mathrm{mm})$ & CV (\%) & \# & CV (\%) \\
\hline \multirow{3}{*}{150} & Control & $20,66 a$ & \multirow{3}{*}{7,16} & $3,46 \mathrm{a}$ & \multirow{3}{*}{5,49} & 9 & \multirow{3}{*}{7,77} \\
\hline & Grupo 1 & $19,30 \mathrm{a}$ & & $3,33 a$ & & 9 & \\
\hline & Grupo 2 & $20,21 \mathrm{a}$ & & $3,49 \mathrm{a}$ & & 10 & \\
\hline \multirow{3}{*}{210} & Control & $27,02 \mathrm{a}$ & \multirow{3}{*}{9,24} & $4,33 \mathrm{a}$ & \multirow{3}{*}{5,57} & 11 & \multirow{3}{*}{9,03} \\
\hline & Grupo 1 & $25,42 \mathrm{a}$ & & $3,99 \mathrm{a}$ & & 12 & \\
\hline & Grupo 2 & $28,12 \mathrm{a}$ & & $4,33 \mathrm{a}$ & & 12 & \\
\hline \multirow{3}{*}{270} & Control & $35,00 \mathrm{a}$ & \multirow{3}{*}{10,39} & $4,77 \mathrm{ab}$ & \multirow{3}{*}{5,91} & 13 & \multirow{3}{*}{7,88} \\
\hline & Grupo 1 & $32,20 \mathrm{a}$ & & $4,41 \mathrm{~b}$ & & 14 & \\
\hline & Grupo 2 & $35,038 \mathrm{a}$ & & $4,93 \mathrm{a}$ & & 13 & \\
\hline
\end{tabular}

Letras diferentes en cada columna indican diferencias significativas (Tukey, $P<0,05$ ).

Different letters in each column indicate significant differences (Tukey, $P<0.05$ ).

Cuadro 4. Masa seca, relación raíz/parte aérea, área foliar, razón del área foliar (RAF) y área foliar especifica (AFE) de plántulas de Aniba rosaeodora, originadas de los tratamientos químico (grupo 1) y biológico (grupo 2) a los 150, 210 y 270 días.

Dry mass, root/aerial part relation, leaf area, relation of the leaf area (RAF), and specific leaf area (AFE) of seedlings of Aniba rosaeodora, originated from seeds treated with chemical insecticide (group 1) and from biological treatment with Beauveria bassiana (group 2) at 150,210 and 270 days.

\begin{tabular}{|c|c|c|c|c|c|c|c|c|}
\hline \multirow{2}{*}{$\begin{array}{l}\text { Edad } \\
\text { (días) }\end{array}$} & \multirow{2}{*}{ Tratamiento } & \multicolumn{3}{|c|}{ Masa seca } & \multirow{2}{*}{$\begin{array}{c}\text { Relación raíz/ } \\
\text { parte aérea }\left(\mathrm{g} \mathrm{g}^{-1}\right)\end{array}$} & \multirow{2}{*}{$\begin{array}{l}\text { Área foliar } \\
\quad\left(\mathrm{cm}^{2}\right)\end{array}$} & \multirow{2}{*}{$\begin{array}{c}\mathrm{RAF} \\
\left(\mathrm{cm}^{2} \mathrm{~g}^{-1}\right)\end{array}$} & \multirow{2}{*}{$\begin{array}{l}\mathrm{AFE} \\
\left(\mathrm{cm}^{2}\right)\end{array}$} \\
\hline & & Raíz & Hoja & Tallo & & & & \\
\hline \multirow{3}{*}{150} & Control & $0,55 \mathrm{a}$ & $1,03 \mathrm{a}$ & $0,50 \mathrm{a}$ & $0,35 \mathrm{a}$ & $157,70 \mathrm{a}$ & $78,74 \mathrm{ab}$ & $156,00 \mathrm{ab}$ \\
\hline & Grupo 1 & $0,63 \mathrm{a}$ & $1,26 \mathrm{a}$ & $0,63 a$ & $0,33 \mathrm{a}$ & $149,62 \mathrm{a}$ & $59,33 b$ & $126,11 \mathrm{~b}$ \\
\hline & Grupo 2 & $0,43 \mathrm{a}$ & $1,09 \mathrm{a}$ & $0,46 \mathrm{a}$ & $0,27 \mathrm{a}$ & $197,30 \mathrm{a}$ & $99,88 \mathrm{a}$ & $169,06 \mathrm{a}$ \\
\hline \multirow{3}{*}{210} & Control & $1,75 \mathrm{a}$ & $2,28 \mathrm{a}$ & $1,20 \mathrm{a}$ & $0,57 \mathrm{a}$ & $325,83 a$ & $64,91 \mathrm{a}$ & $144,04 \mathrm{a}$ \\
\hline & Grupo 1 & $1,33 \mathrm{a}$ & $2,60 \mathrm{a}$ & $1,33 \mathrm{a}$ & $0,37 \mathrm{a}$ & $383,92 \mathrm{a}$ & $73,02 \mathrm{a}$ & $147,80 \mathrm{a}$ \\
\hline & Grupo 2 & $1,47 \mathrm{a}$ & $2,91 \mathrm{a}$ & $1,42 \mathrm{a}$ & $0,36 \mathrm{a}$ & $347,55 \mathrm{a}$ & $60,61 \mathrm{a}$ & $136,04 \mathrm{a}$ \\
\hline \multirow{3}{*}{270} & Control & $1,61 \mathrm{a}$ & $2,78 \mathrm{a}$ & $1,28 \mathrm{a}$ & $1,40 \mathrm{a}$ & $479,05 \mathrm{a}$ & $85,44 a$ & $172,17 \mathrm{a}$ \\
\hline & Grupo 1 & $1,53 \mathrm{a}$ & $2,92 \mathrm{a}$ & $1,35 \mathrm{a}$ & $1,36 \mathrm{a}$ & $474,79 a$ & $83,62 \mathrm{a}$ & $162,53 \mathrm{a}$ \\
\hline & Grupo 2 & $1,71 \mathrm{a}$ & $2,82 \mathrm{a}$ & $1,59 a$ & $1,39 \mathrm{a}$ & $454,75 \mathrm{a}$ & $76,12 \mathrm{a}$ & $163,41 \mathrm{a}$ \\
\hline
\end{tabular}

En cada columna, letras diferentes indican diferencias significativas (Tukey, $P<0,05$ ).

Different letters in each column indicate significant differences (Tukey, $P<0.05$ ). 
Pigmentos cloroplastos. Los resultados evaluados de los parámetros de pigmentos de clorofila y carotenoides de las plántulas de A. rosaeodora presentaron un padrón similar que puede estar relacionado a las condiciones de su desarrollo en vivero. Además, indican que la especie no altera su sistema de producción de pigmentos de clorofila en relación al tratamiento aplicado a las semillas, y sugieren que no existe influencia en el desarrollo inicial de plántulas (figura 3).

\section{DISCUSIÓN}

La situación actual de $A$. rosaeodora relacionada a la falta del material biológico, obliga a los investigadores a buscar nuevas alternativas que permitan aumentar la producción de plántulas destinadas a plantaciones forestales, recuperación de áreas degradas, como también la incorporación de la especie en los sistemas agroforestales. Los tratamientos en las semillas alcanzaron los objetivos, aumentando los índices de germinación, dentro de los parámetros establecidos por Sampaio et al. (2003), indicando que el proceso inicial varía entre 17 y 128 días en condiciones de laboratorio $\left(25\right.$ a $\left.35^{\circ} \mathrm{C}\right)$. Las semillas no presentan características de dormancia, a pesar de que el tegumento tiene cierta resistencia física a la emisión radicular. Por otro lado, las semillas colectadas en el suelo, al ser embebidas por más tiempo, tienden a reducir su germinación, efecto que fue invertido en semillas colectadas de la copa, factor que probablemente puede estar relacionado al estado de las semillas, siendo que al ser colectadas todavía estaban en la fase inmadura.

El proceso de imbibición influenció positivamente la germinación, en ese periodo, los líquidos son absorbidos por la semilla y eso provoca la hidratación de los tejidos, ocurriendo la intensificación de la respiración y las actividades metabólicas, que resultan en el fornecimiento de energía y crecimiento por parte del embrión (De Castro y Hillhorst 2004). En contra partida, las semillas colectadas en el suelo fueron liberadas por la planta matriz debido al proceso natural de dispersión de semillas, por tanto, inician el proceso de desarrollo con antecedencia y embeberlas puede interferir en el proceso de germinación.

La utilización del insecticida adecuado puede proteger la salud de las plantas, aumentar el crecimiento, reducir el número de síntomas relacionadas a la patogénesis y conseguir una mitigación del estrés (Elbert et al. 2008). El crecimiento de las especies vegetales es un proceso complejo, sin embargo, se considera como una técnica precisa para evaluar el desarrollo y la fisiología, que también depende de la edad, partes de la planta y de la interacción con los factores ambientales (Campos et al. 2008). En ese sentido, estudios de Morais et al. (2009) sobre el desarrollo inicial basado en la protección externa de las semillas de $A$. rosaeodora con insecticidas, determinó el crecimiento de las plántulas con 11 y $12 \mathrm{~cm}$ de altura a los 180 días de edad. En el presente estudio, los tratamientos de imbibición determinaron que las plántulas alcanzaron $20 \mathrm{~cm}$ de altura a los 150 días de edad, factor que podría estar relacionado al aceleramiento en el proceso de germinación, además de mantener uniformidad en la producción de biomasa, posiblemente relacionada a la capacidad de luz, absorción de agua y sustrato.

En relación a la fisiología de las plantas, los tratamientos de semillas a través de la imbibición, pueden alterar el transporte de electrones en el foto sistema, influyendo en las tasas líquidas de fotosíntesis o perjudicando la conductancia estomática (Henry et al. 2011). Esa definición coincide con el desarrollo de las plántulas originadas del tratamiento biológico, que presentaron los valores más bajos en las variables de fotosíntesis líquida, conductancia estomática y transpiración, en contra partida, estas variables permitieron determinar que el balance entre la pérdi-

Cuadro 5. Intercambio de gases y características fotosintéticas con desvió padrón de plántulas de Aniba rosaeodora con 8 meses de edad, originadas de semillas con tratamiento químico (grupo 1) y tratamiento biológico con Beauveria bassiana (grupo 2).

Gas exchange and photosynthetic characteristics with standard deviation of the seedlings of Aniba rosaeodora with eight months of age, originated from seeds treated with chemical insecticide (group 1) and from biological treatment with Beauveria bassiana (group 2).

\begin{tabular}{lccc}
\hline \multicolumn{1}{c}{ Variables } & Control & Grupo 1 & Grupo 2 \\
\hline $\mathrm{A}\left[\mu \mathrm{mol} \mathrm{CO} \mathrm{C}^{-2} \mathrm{~s}^{-1}\right]$ & $6,25 \pm 0,83 \mathrm{a}$ & $5,31 \pm 1,18 \mathrm{ab}$ & $3,70 \pm 1,44 \mathrm{~b}$ \\
$\mathrm{Rd}\left[\mu \mathrm{mol} \mathrm{CO} \mathrm{m}^{-2} \mathrm{~s}^{-1}\right]$ & $0,35 \pm 0,33 \mathrm{a}$ & $0,71 \pm 0,24 \mathrm{a}$ & $0,44 \pm 0,17 \mathrm{a}$ \\
$\mathrm{gs}\left[\mathrm{mmol} \mathrm{H} \mathrm{O} \mathrm{m}^{-2} \mathrm{~s}^{-1}\right]$ & $0,11 \pm 0,02 \mathrm{a}$ & $0,09 \pm 0,04 \mathrm{~b}$ & $0,04 \pm 0,02 \mathrm{~b}$ \\
$\mathrm{E}\left[\mathrm{mmol} \mathrm{H} \mathrm{O} \mathrm{m}^{-2} \mathrm{~s}^{-1}\right]$ & $2,07 \pm 0,23 \mathrm{a}$ & $1,71 \pm 0,59 \mathrm{a}$ & $0,78 \pm 0,46 \mathrm{~b}$ \\
$\mathrm{EUA}\left[\mathrm{mmol} \mathrm{CO}_{2} \mathrm{~mol}^{-1} \mathrm{H}_{2} \mathrm{O}\right]$ & $3,02 \pm 0,18 \mathrm{~b}$ & $3,34 \pm 0,76 \mathrm{~b}$ & $4,74 \pm 0,89 \mathrm{a}$ \\
\hline
\end{tabular}

Fotosíntesis neta (A), Respiración en lo oscuro (Rd), Conductancia estomática (gs), Transpiración (E) y Eficiencia en el uso de agua (EUA). En cada columna, letras diferentes indican diferencias significativas (Tukey, $P<0,05$ ).

Net photosynthesis (A), dark respiration (Rd), stomata conductance (gs), transpiration (E) and water use efficiency (EUA). Different letters in each column indicate significant differences (Tukey, $P<0.05$ ). 
da de agua y la absorción del $\mathrm{CO}_{2}$ presenta una estrategia positiva de eficiencia en el uso del agua. En ese sentido, la evaluación de los indicadores fisiológicos asociados a la respuesta de las plántulas originadas de semillas con tratamiento inicial se torna una herramienta altamente importante.
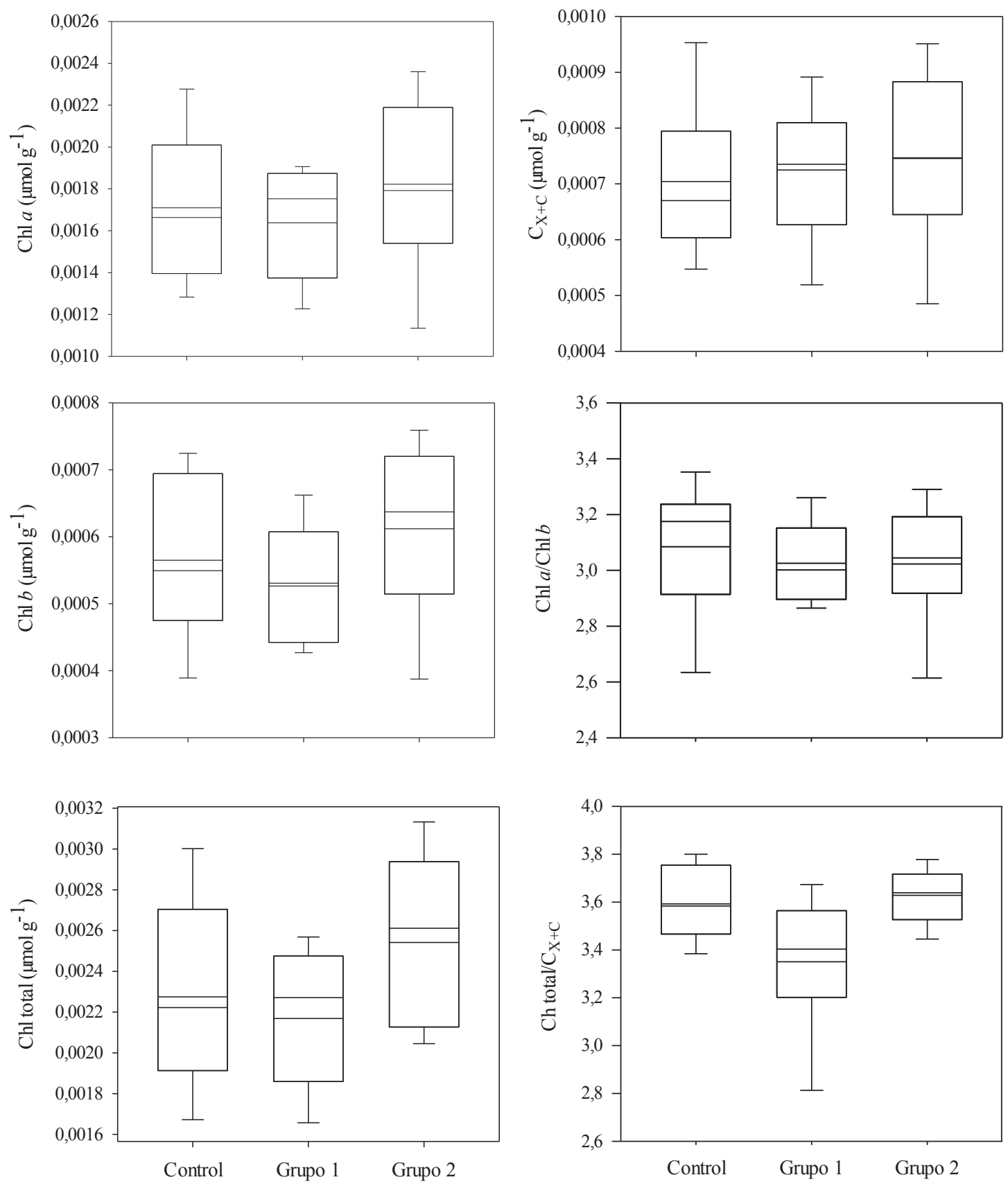

Figura 3. Box-plot del contenido de pigmentos de clorofila en la base de la masa foliar $(\mathrm{n}=10)$ de plántulas de Aniba rosaeodora, originadas de los tratamientos químico (grupo 1) y biológico (grupo 2). Clorofila $a(\mathrm{Chl} a)$, clorofila $b(\mathrm{Chl} b)$, clorofila total (Chl $a+$ $\mathrm{Chl} b)$, carotenoides $\left(\mathrm{C}_{\mathrm{x}+\mathrm{c}}\right)$ clorofila $a / b(\mathrm{Chl} a / \mathrm{Chl} b)$ y clorofila total/carotenoides $\left(\mathrm{Chl}\right.$ total $\left./ \mathrm{C}_{\mathrm{x}+\mathrm{c}}\right)$.

Box-plot with the contents of pigments chlorophyll leaf mass $(\mathrm{n}=10)$ of seedlings of Aniba rosaeodora, originated from seeds treated with chemical insecticide (group 1) and from biological treatment with Beauveria bassiana (group 2). Chlorophyll $a$ (Chl $a$ ), chlorophyll $b$ (Chl $b$ ), total chlorophyll $(\mathrm{Chl} a+\mathrm{Chl} b)$, carotenoids $\left(\mathrm{C}_{\mathrm{x}+\mathrm{c}}\right)$, chlorophyll $a / b(\mathrm{Chl} a / \mathrm{Chl} b)$ and total chlorophyll/carotenoids $\left(\mathrm{Chl}\right.$ total/ $\left.\mathrm{C}_{\mathrm{x}+\mathrm{c}}\right)$. 
que afectan las características fotosintéticas. En el caso de A. rosaeodora, las plántulas analizadas no presentaron variaciones de clorofila $a, b$ y carotenoides, por tanto, no presentan estrés ambiental. De esa manera, se puede indicar que los tratamientos químico y biológico aplicados a semillas de A. rosaeodora no alteran su sistema de producción de pigmentos de clorofila en plántulas.

\section{CONCLUSIONES}

Las características y estado fisiológico de las semillas infectadas de $A$. rosaeodora colectadas de la copa del árbol, presentaron capacidad de resistencia después de sometidas a la imbibición en insecticida, evitando el desenvolvimiento de larvas de $H$. odoratus y en consecuencia, aumentan el número de semillas germinadas, así también, se determina que existe la relación entre los tiempos más prolongados de imbibición con el aumento de los índices de germinación. En el caso de colectar semillas en el suelo, se recomienda limitar los procesos de imbibición en insecticida hasta 48 horas. Por otro lado, visando la protección de semillas, además de aumentar los índices de germinación, se recomienda la aplicación del hongo entomopatógeno $B$. bassiana como tratamiento inicial, en ese sentido, en términos de producción de plántulas, se recomienda trabajar con semillas sin tegumento para acelerar el proceso de germinación. Las evaluaciones de las características fisiológicas analizadas en las plántulas originadas de los tratamientos químico y biológico, permiten determinar que no existen influencias que puedan alterar el desarrollo inicial, por tanto, dadas las condiciones, están aptas para ser destinadas a plantaciones forestales.

\section{AGRADECIMIENTOS}

Al Programa de Pos-Graduación en Biotecnología y Recursos Naturales (MBT-UEA) por el convenio con el Instituto Nacional de Pesquisas de la Amazonia (INPA). Al Laboratorio de Cultivo de Hongos Comestibles (LCFC/ INPA) por las instalaciones para realizar los experimentos, además de la transferencia de la cepa. Al Dr. Geângelo Petene Calvi por las fotografías de Rayos-X. Esta investigación fue financiada por el Consejo Nacional de Desenvolvimiento Científico y Tecnológico (CNPq).

\section{REFERENCIAS}

Arnon DI. 1949. Copper enzymes in isolated choroplasts: Polyphenoloxidase in Beta Vulgaris. Plant Physiology 24:1-15.

Barros AT, J Otea, D Sanson, LD Foil. 2001. Horn fly (Diptera: Muscidae) resistance to organophosphate insecticides. Veterinary Parasitology 96(3):243-256.

Brasil. 2009. Regras para análises de sementes. Brasilia, Brasil. MAPA. 399 p.

Campos MF, EE Ono, CSF Boaro, JD Rodrigues. 2008. Análise de crescimento em plantas de soja tratadas com substâncias reguladoras. Biotemas 21(3):53-63.
De Castro RD, HWM Hilhorst. 2004. Embebição e reativação do metabolismo In Ferreira AG, Borghetti Eds. Germinação do básico ao aplicado. Porto Alegre, Brasil. Artmed. p. 149-162.

Delgado, PAM, B Murcia-Ordoñez. 2011. Hongos entomopatógenos como alternativa para el control biológico. Revista Ambiente \& Água 6(2):77-90.

Embrapa. 2006. Sistema Brasileiro de classificação de solos. Brasilia, Brasil. CNPS. 306 p.

Elbert A, M Haas, B Springer, W Thielert, R Nauen. 2008. Applied aspects of neonicotinoid uses in crop protection. Pest Management Science 64(11): 1099-1105.

Ferreira JMS, AV Teodoro, ASN Junior, EC Guzzo. 2014. Manejo integrado da broca-do-olho-do-coqueiro Rhynchophorus palmarum L (Coleoptera: Curculionidae). Empresa Brasileira de Pesquisas Agropecuarias (Comunicado Técnico). Consultado 11 dic. 2017. Disponible en https://www.researchgate.net/publication/279763155_Ferreira_et_al_2014.

Gonçalves JFC, DCS Barreto, UMS Junior, AV Fernandes, PTB Sampaio, MS Buckeridge. 2005. Growth, photosynthesis and stress indicators in Young rosewood plants (Aniba rosaeodora Ducke) under different light intensities. Brazilian Journal of Plant Physiology 17(3): 325-334.

Gonçalves JFC, AV Fernandes, CEM Silva, UMS Junior, PTB Sampaio. 2016. Características ecofuncionais de sementes e plantas jovens de Aniba rosaeodora. In Sampaio PTB, HS Pereira eds. Uso e conservação do Pau-rosa. Manaos, Brasil. Ufam. p. 77-100.

Green J, DM Newber. 2001. Light and seed size affect establishment of grove-forming ectomycorrhizal rain forest tree species. New Phytologist 151: 271-289.

Hendry GAF, AH Price. 1993. Stress indicators: Chlorophylls and carotenoids. In Hendry GAF, JP Grime eds. Methods in comparative plant ecology. Londres, Inglaterra. p. 123-127.

Henry R.S, WG Johnson, KA Wise. 2011. The impact of a fungicide and na insecticide on soybean growth, yield, and profitability. Crop Protection 30: 1629-1634.

INMET (Instituto Nacional de Meteorologia, BR). 2017. Instituto Nacional de Metereologia- Ministério da Agricultura, Pecuária e Abastecimento. Consultado 5 dic. 2017. Disponible en http://www.inmet.gov.br/portal/index.php?r=home/ page \& page $=$ clima.

Kaewkham T, RK Hynes, B Siri. 2016. The effect of accelerated seed ageing on cucumber germination following seed treatment with fungicides and microbial biocontrol agentes for managing gummy stem blight by Didymella bryniae. Biocontrol Science and Technology 26(8):1048-1061.

Krainovic PM, DRA de Almeida, D Desconci, VF da VeigaJúnior, PTB Sampaio. 2017. Sequential management of comercial rosewood (Aniba rosaeodora Ducke) plantations in Central Amazonia: Seeking sustainable models for essential oil production. Forests 8(12):438.

Leite L.G, FM Tavares, CMA Ginarte, LC Carregari, FA Batista. 2006. Nematóides entomopatogênicos no controle de pragas. In Pinto AS, DE Nava, M Rossi, DT Malerbo-Souza eds. Controle biológico na prática. São Paulo, Brasil. p. 45-53.

Lorenzi H. 1998. Árvores brasileiras: Manual de identificação e cultivo de plantas árboreas nativas do Brasil. São Paulo, Brasil. Instituto Plantarum. 352p.

Marenco RA, JF Gonçalves, G Vieira. 2001. Leaf gas exchange and carbohydrates in tropical trees differing in successional status in two light environments in central Amazonia. Tree 


\section{Physiology 21: 1311-1318.}

Melgarejo LM. 2010. Experimentos en fisiología vegetal. Bogotá, Colombia. Unal. 277 p.

Morais JW, JAM Figueira, PTB Sampaio. 2009. Eficiência de inseticidas no controle de pragas em sementes e mudas de Pau-rosa (Aniba rosaeodora Ducke), em viveiros, Manaus, Amazonas. Acta Amazonica 36(3): 533-538.

Sampaio PT, ID Ferraz, JL Camargo. 2003. Manual de sementes da Amazônia. Amazonas, Brasil. Projeto Jacarandá. 7 p.
Spironello, WR, PTB Sampaio, B Ronchi-Teles. 2004. Produção e predação de frutos de Aniba rosaeodora Ducke var. amazonica Ducke (Lauraceae) em sistema de plantio sob floresta de terra firme na Amazônia Central. Acta Botánica Brasileira 18(4): 801-807.

Vanin, AS, F Gaiger. 2005. A new spermophagous species of Heilipus Germar from the Amazonian Region (Coleoptera, Curculionidae, Molytinae). Revista Brasileira de Entomologia 49(2): 240-244.

Recibido: 02/01/18

Aceptado: 06/06/18 